\title{
Autoxidation of Fish Oil Blended with Rice Bran Oil
}

\author{
Shigeo Nakajima ${ }^{1}$, Marie Takai ${ }^{1}$, Chieko Hayashi ${ }^{1}$, Takuo Tsuno ${ }^{1}$ and Yasushi Endo ${ }^{2 *}$ \\ ${ }^{1}$ Tsuno Food Industrial Co. Ltd. 94 Shinden, Katsuragi, Ito, Wakayama 649-7194, JAPAN \\ ${ }^{2}$ School of Bioscience and Biotechnology, Tokyo University of Technology, Tokyo 192-0982, JAPAN
}

\begin{abstract}
Effects of rice bran oil on the oxidative and flavor stability of fish oil were investigated by the gas liquid chromatography-head space method. When fish oil blending with different ratio of rice bran oils was oxidized at room temperature in the dark, volatile compounds produced during autoxidation was measured by gas liquid chromatography. The amounts of volatile compounds were decreased with increased the ratio of blended rice bran oil as well as peroxide value. The level of propanal and acrolein which gave unpleasant flavor was also decreased with increased the ratio of blended rice bran oil. Especially, the level of propanal and acrolein and peroxide value were remarkably decreased when blending more than $\mathbf{7 5 \%}$ of rice bran oil. Blending of rice bran oil improved the oxidative and flavor stabilities of fish oil.
\end{abstract}

Key words: acrolein, autoxidation, fish oil, polyunsaturated fatty acid, propanal, rice bran oil

\section{Introduction}

n-3 Polyunsaturated fatty acids (PUFAs) such as eicosapentaenoic acid (EPA, 20:5) and docosahexaenoic acid (DHA, 22:6) contained in fish oil have important physiological functions such as antithrombotic, cholesterol depressing and anti-allergenic properties ${ }^{1-4)}$. Thus, fish oils such as sardine, tuna and mackerel oils are often used as dietary supplements and functional foods. However, n-3 PUFAs are susceptible to autoxidation and thermal oxidation. Oxidative degradation products of n-3 PUFAs, especially low molecular compounds give rise to off-flavor and reduce the quality of fish oil. This instability of n-3 PUFAs makes application of fish oil to dietary supplements and functional foods difficult. Therefore, stabilization of fish oil is necessary for them.

In general, antioxidants such as tocopherols are added to fish oils rich in n-3 PUFAs, to improve the oxidative stability. However, addition of antioxidants is not always satisfied for retarding the production of off-flavor from fish oils during storage. Blending other fats and oils to fish oils has been attempted to maintain the quality of fish oils during storage. Shiota et $a l .{ }^{5}$ tried blending butter to fish oils to improve the oxidative stability of fish oils. When Saga et $a l{ }^{6)}$ assessed the oxidative stability of fish oils blended with crude plant oils such as camellia oil and oat oil by the Schaal oven weight gain test and by the rancimat method, crude oat oil increased the oxidative stability of fish oil. However, these fats and oils may affect the physiological properties and give unpleasant flavor to fish oil.

Rice bran oil(RBO) which is a vegetable oil extracted from the germ and bran of the rice grain is eaten and favorable throughout the world. RBO has specific characteristics that natural antioxidants such as vitamin $\mathrm{E}$ (tocopherols and tocotrienols) and $\gamma$-oryzanol are richer than other vegetable oil ${ }^{7)}$. Thus, RBO is very stable for the oxidation and thermal degradation. Moreover, RBO may provide masking ability to give sweet and pleasant flavor based on vanillin derived from $\gamma$-oryzanol. Blending of RBO may improve the oxidative and flavor stabilities of fish oil rich in n-3 PUFAs. In this study, fish oil blended with different ratio of RBO was autoxidized and then volatile compounds produced during autoxidation were measured by gas liquid chromatography, to find the optimal blending ratio of RBO.

\section{Materials and Methods}

2.1 Rice bran oil and fish oil

Refined RBO were provided by Tsuno Food Industrial Co. Ltd(Wakayama, Japan). Refined fish oil was provided by Maruha Nichiro, Ltd. (Tokyo, Japan).

\subsection{Fatty acid composition and tocopherol content}

Gas liquid chromatographic method $\left(2.4 .2 .3_{-2013}\right)^{8)}$ and high performance liquid chromatographic method (2.4.10. ${ }_{2013}{ }^{9)}$ described in the "Standard Methods for the Analysis of Fats, Oils and Related Materials" published by The Japan Oil Chemists' Society(2013)was employed to measure the fatty acid composition and tocopherol contents of fish oil and RBO, respectively.

*Correspondence to: Yasushi Endo, School of Bioscience and Biotechnology, Tokyo University of Technology, Tokyo 192-0982, JAPAN

E-mail: endo@bs.teu.ac.jp

Accepted March 6, 2017 (received for review February 6, 2017)

Journal of Oleo Science ISSN 1345-8957 print / ISSN 1347-3352 online

http://www.jstage.jst.go.jp/browse/jos/ http://mc.manusriptcentral.com/jjocs 


\subsection{Autoxidation test}

Sixty grams of fish oil blended with $0,13,25,30,50,60$, 75,90 and $100 \%$ of RBO were put in a 140 mL-glass vessel and then they were stored at room temperature in the dark. Periodically, volatile compounds and hydroperoxides produced from fish oils blended with RBO were measured during autoxidation.

\subsection{Peroxide value (PV)}

The titration method $\left(2 \cdot 5.2^{2} 1_{-2013}\right){ }^{10)}$ to determine peroxide value (PV) described in the "Standard Methods for the Analysis of Fats, Oils and Related Materials" published by The Japan Oil Chemists' Society(2013) was employed.

\subsection{Volatile compounds ${ }^{11)}$}

Two grams of oxidized oils was put in a 20 mL-glass vial, and it was sealed with a metal cap. The vial was heated at $55^{\circ} \mathrm{C}$ for $10 \mathrm{~min}$, and then a $1.0 \mathrm{~mL}$ head space gas was injected into the gas chromatograph.

HERACLES II gas chromatograph (Alpha M.O.S Japan) equipped with a FID detector was used. For the columns, $18 \mathrm{~m} \times 0.18 \mathrm{~mm}$ i.d. DB-5 or WAX fused silica capillary column was used. The injector temperature was $220^{\circ} \mathrm{C}$ and detector temperature was $250^{\circ} \mathrm{C}$. The column was held at $40^{\circ} \mathrm{C}$ and then programmed to $250^{\circ} \mathrm{C}$ at $3^{\circ} \mathrm{C} / \mathrm{s}$ after the injection of a head space gas. Hydrogen gas was used as a carrier gas.

Volatile compounds were identified by the retention time of authentic standards and gas chromatography-mass spectrometry.

Sensory evaluation was also performed for stored fish oils blended with RBO during storage by 4 to 5 panelists. Panelists compared the intensity of off-flavor of fish oils blended with RBO after autoxidation.

Experiments were performed in triplicate. All data described the average, because the relative standard deviation was below 10\%. Data were statistically analyzed by analysis of variance (ANOVA) . Duncan's multiple range test was used to determine the differences among samples.

\section{Results and Discussion}

\subsection{Fatty acid composition and vitamin E content}

Table 1 shows the fatty acid composition of fish oil and RBO. Fish oils contained 6\% EPA and 27\% DHA as n-3 PUFAs. On the other hand, RBO contained $43 \%$ oleic acid and $36 \%$ linoleic acid as major fatty acids. Table 2 shows the vitamin $\mathrm{E}$ (tocopherols and tocotrienols) contents of fish oil and RBO. Fish oil contained high amounts (123 $\mathrm{mg} / 100 \mathrm{~g}$ ) of tocopherols, because tocopherols were added as food additives. RBO contained $75 \mathrm{mg} / 100 \mathrm{~g}$ tocopherols and $55 \mathrm{mg} / 100 \mathrm{~g}$ tocotrienols. Total vitamin $\mathrm{E}$ content in fish oil was same as that in RBO. RBO also contained 1.5\% $\gamma$-oryzanol as a natural antioxidant. $\gamma$-Oryzanol consisted of $36 \%$ cycloartenyl ferulate, 45\% 24-methylenecycloartanyl ferulate, $14 \%$ campesteryl ferulate and $4 \% \beta$-sitosteryl ferulate.

\subsection{Peroxide value}

Figure 1 shows the peroxide value (PV) of fish oils blended with RBO during autoxidation. PV of fish oils blended with RBO was increased during autoxidation at room temperature, although unoxidized oils had very low PV (less than $1 \mathrm{meq} / \mathrm{kg}$ ). Increase rate of PV was higher with the ratio of fish oil, because fish oil had considerable amounts of PUFAs. PV of fish oil and fish oil blended with $13 \%$ RBO exceeded 30 meq/kg after 21 days, while those of fish oils blended with $75 \%$ and $90 \%$ RBO besides RBO were less than 5 meq/kg after 28 days. When fish oils were blended with more than $75 \%$ RBO, the production of hydroperoxides was remarkably retarded during autoxidation at room temperature. Fish oils blended with $75 \%$ and $90 \%$ RBO were oxidatively stable, although they contained $1.5 \%$ EPA and 6.8\% DHA, and 0.6\% EPA and 2.7\% DHA, respectively. From these results, blending of more than $75 \%$ RBO to fish oil was effective for improving the oxidative stability of fish oil rich in n-3 PUFAs.

\subsection{Volatile compounds}

Volatile compounds in head space gas from fish oil blended with RBO were measured by gas liquid chromatog-

Table 1 Major fatty acids of fish oil and rice bran oil(RBO).

\begin{tabular}{lcccccccc}
\hline Fatty acid (\%) & $16: 0$ & $16: 1$ & $18: 0$ & $18: 1$ & $18: 2$ & $18: 3$ & $20: 5$ & $22: 6$ \\
\hline Fish oil & 20 & 11 & 5 & 25 & 3 & 3 & 6 & 27 \\
Rice bran oil & 17 & - & 2 & 43 & 36 & 1 & - & - \\
\hline
\end{tabular}

Table 2 Tocopherols and tocotrienols contents of fish oil and rice bran oil(RBO).

\begin{tabular}{lccccccccccc}
\hline $\begin{array}{l}\text { Vitamin E } \\
(\mathrm{mg} / 100 \mathrm{~g})\end{array}$ & $\alpha$-Toc & $\beta$-Toc & $\gamma$-Toc & $\delta$-Toc & Total Toc & $\alpha$-Tri & $\beta$-Tri & $\gamma$-Tri & $\delta$-Tri & Total Tri & Total VE \\
\hline Fish oil & 24.1 & 1.6 & 74.8 & 22.3 & 122.9 & - & - & - & - & - & 122.9 \\
Rice bran oil & 47.7 & 1.8 & 19.7 & 5.4 & 74.6 & 25.4 & 1.8 & 27.2 & 0.8 & 55.1 & 129.7 \\
\hline
\end{tabular}

VE, vitamin E; Toc, tocopherol; Tri, tocotrienol 


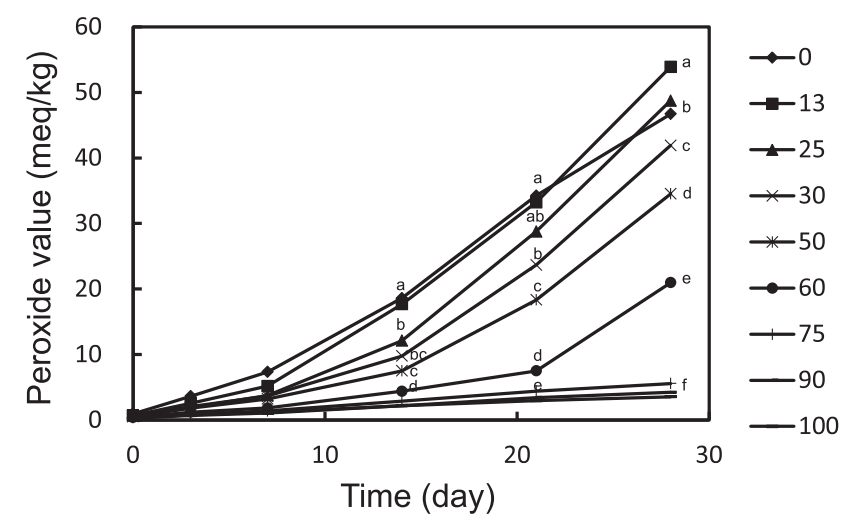

Fig. 1 Peroxide value (PV) of fish oils blended with RBO during autoxidation at room temperature. Values with different letters are significantly different $(p<$ $0.05)$.

raphy (GLC) during autoxidation at room temperature. Gas chromatograms of volatile compounds from fish oil and fish oil blended with $75 \%$ of RBO after autoxidation for 28 day are shown in Figs. 2 and 3, respectively. As shown in Fig. 2, many varieties of volatile compounds were detected in oxidized fish oil. Major volatile compounds included carbonyl compounds such as acetaldehyde, propanal, acrolein, butanal besides ethanol as an alcohol. These volatile compounds could be derived from n-3 PUFAs such as EPA and DHA besides $\alpha$-linolenic acid (18:3) contained in vegetable oils ${ }^{12)}$. Among volatile compounds, carbonyl compounds could be responsible for off-flavor of oxidized fish oil, because they had very low threshold (ppb level).

As shown in Fig. 3, several volatile compounds were also detected in oxidized fish oil blended with $75 \%$ of RBO after autoxidation for 28 days, although their peak intensities were low. A gas chromatogram of volatile compounds from oxidized fish oil blended with $75 \%$ RBO was quite different from that from oxidized fish oil. Peaks due to propanal and acrolein which were major volatile compounds from oxidized fish oil, as shown in Fig. 2 were very small. That is, volatile compounds derived from n-3 PUFAs such as EPA and DHA were not almost produced during autoxidation of fish oil blended with $75 \%$ RBO. Major volatile compounds included acetone, acetaldehyde, butanal and pentanal in oxidized fish oil blended with $75 \%$ RBO, although their peak intensities were very low. Probably, they were mainly produced from linoleic acid (18:2) contained in RBO during autoxidation.

Among volatile compounds, propanal and acrolein had very low threshold and irritating odor. Moreover, their levels were much higher among volatile compounds produced from n-3 PUFAs in fish oil and they gave rise to offflavor. Therefore, the levels of propanal and acrolein were estimated during autoxidation of fish oils blended with RBO. Figures 4 and 5 show the levels of propanal and acrolein produced during autoxidation of fish oils blended with $\mathrm{RBO}$ at room temperature in the dark, respectively. As shown in Fig. 4, the level of propanal from fish oil was increased during autoxidation, although that from unoxidized fish oil was $4.6 \mathrm{ppb}$. The propanal level was increased to $18.6 \mathrm{ppm}$ in fish oil oxidized for 28 days. On the other hand, fish oils blended with $75 \%$ and $90 \%$ RBO showed approximately 5 ppb propanal after 28 days. Propanal was not almost produced from fish oil blended with more than $75 \%$ RBO during autoxidation at room temperature.

As shown in Fig. 5, the level of acrolein from fish oil was also increased during autoxidation, although that from un-

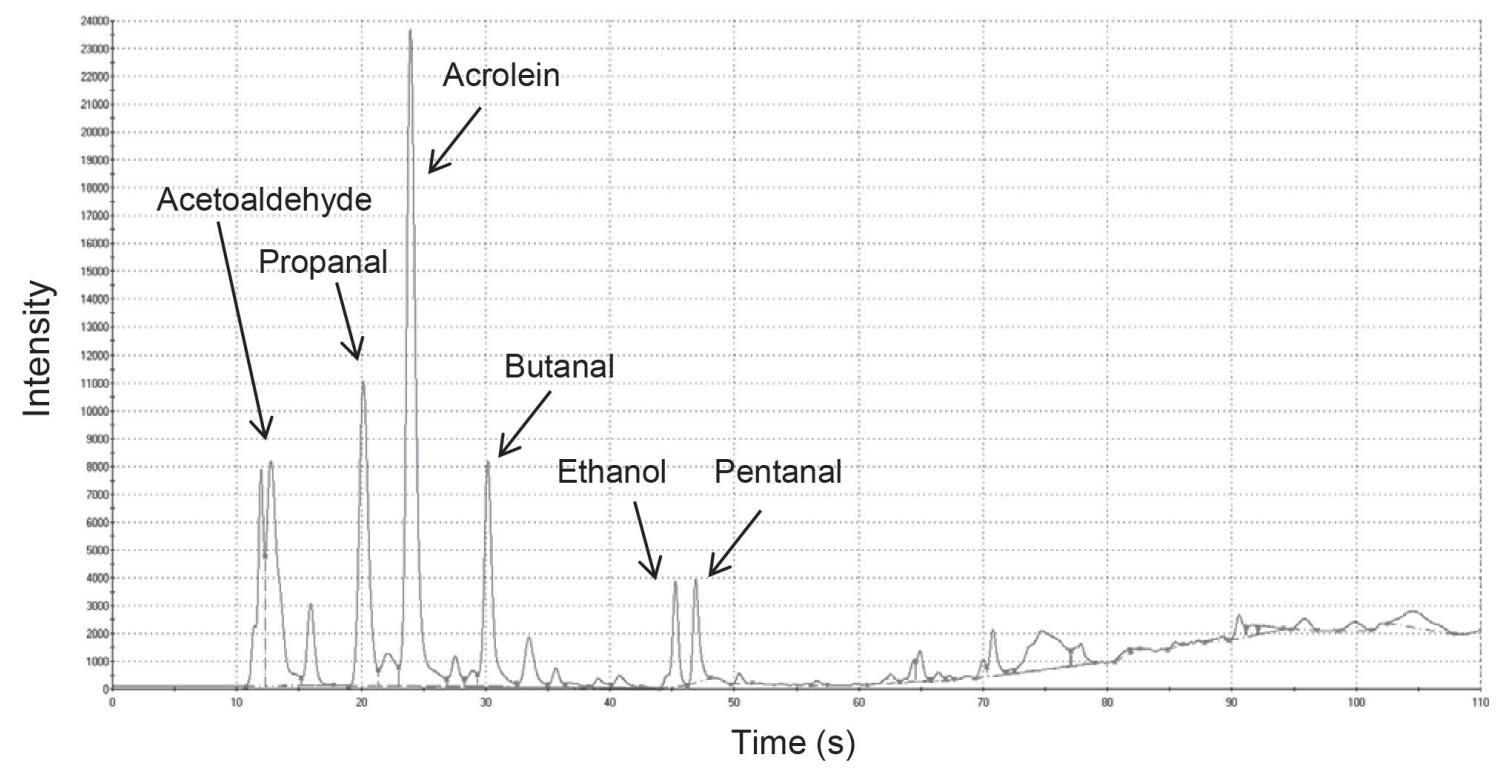

Fig. 2 Gas chromatogram of volatile compounds from fish oil after autoxidation at room temperature for 28 days. 


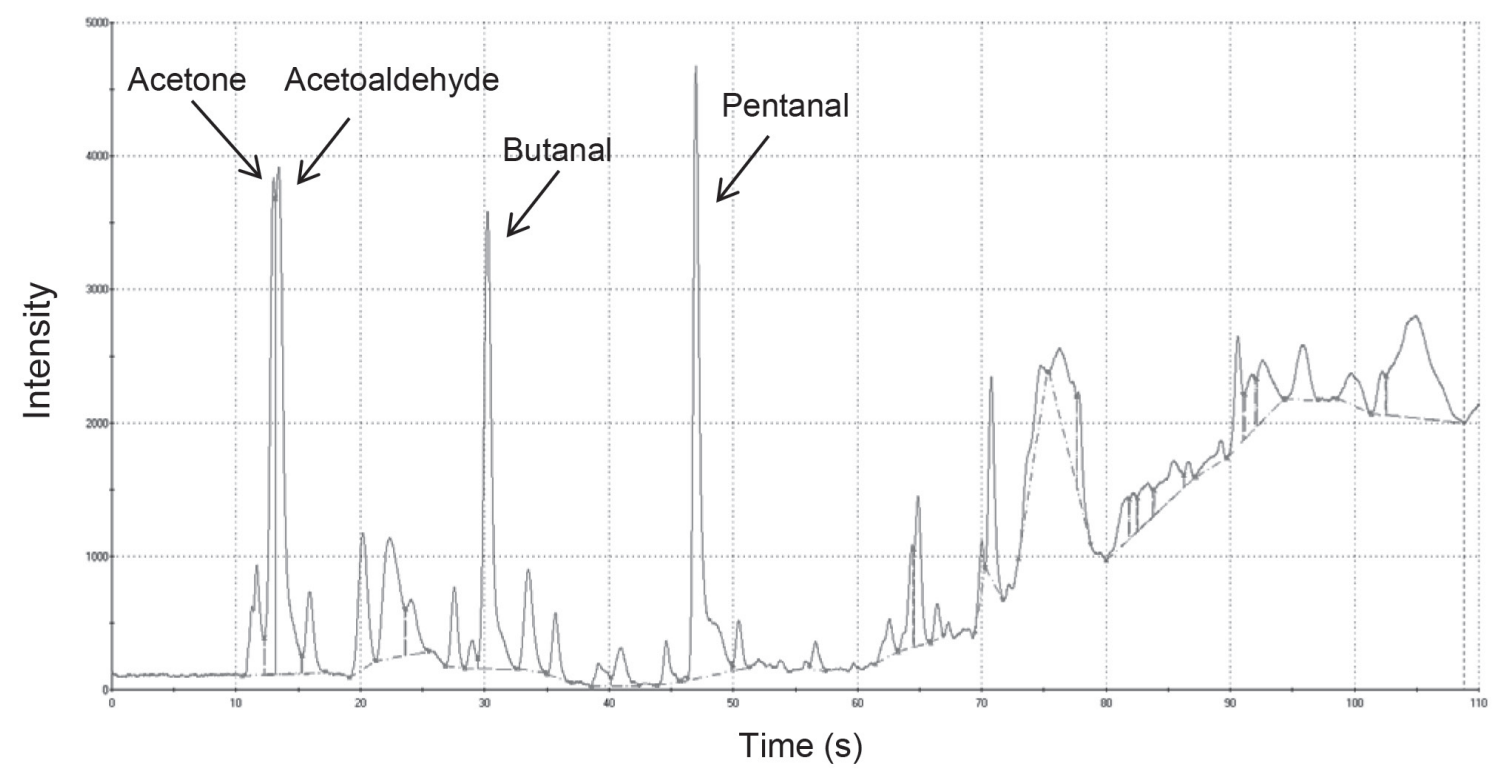

Fig. 3 Gas chromatogram of volatile compounds from fish oil blended with $75 \%$ RBO after autoxidation at room temperature for 28 days.

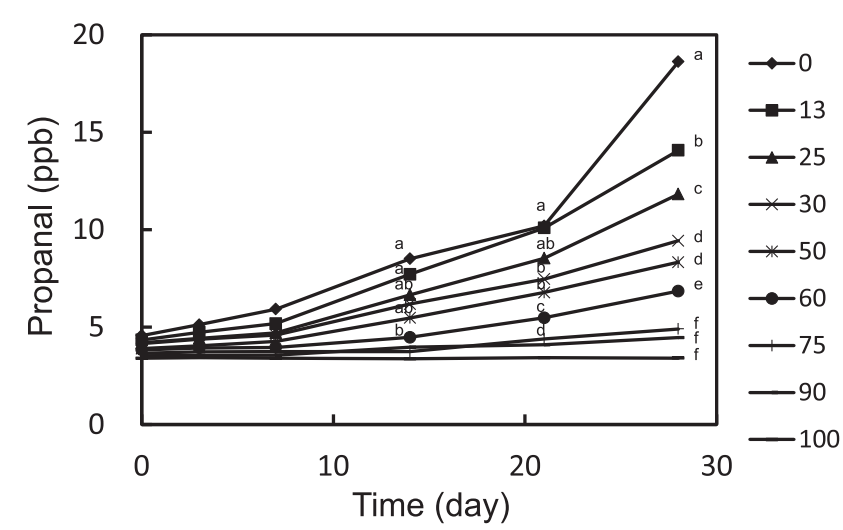

Fig. 4 Propanal produced from fish oils blended with RBO during autoxidation at room temperature. Values with different letters are significantly different $(p<$ $0.05)$.

oxidized fish oil was $4.3 \mathrm{ppb}$. The acrolein level was increased to $61.7 \mathrm{ppb}$ in fish oil oxidized for 28 days. On the other hand, fish oils blended with $75 \%$ and $90 \%$ RBO showed approximately 6 ppb acrolein after 28 days. Acrolein was not almost produced from fish oil blended with more than $75 \%$ RBO during autoxidation at room temperature. The low levels of propanal and acrolein in fish oil blended with more than 75\% RBO could be based on the low level of PV(hydroperoxides), because these carbonyl compounds were produced from hydroperoxides of n-3 PUFAs such as EPA and DHA.

As for the sensory evaluation, fish oil showed unpleasant off-flavor during autoxidation at room temperature, while the intensity of off-flavor from fish oil blended with $75 \%$ or

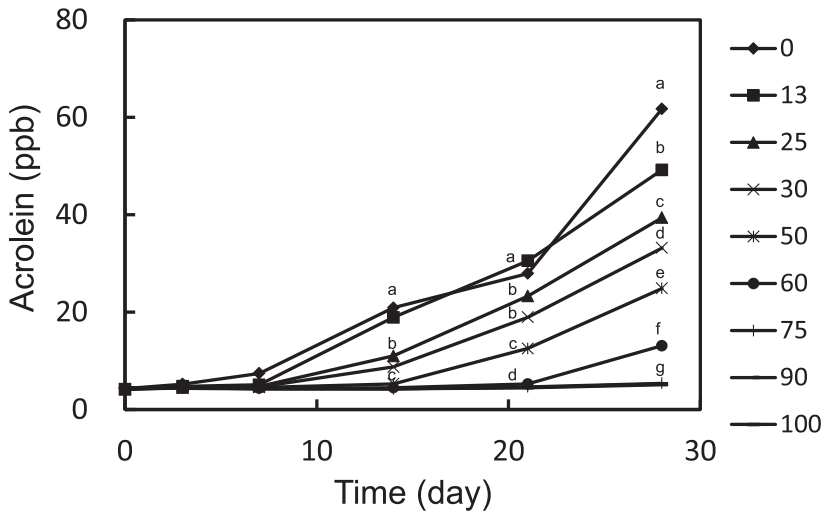

Fig. 5 Acrolein produced from fish oils blended with RBO during autoxidation at room temperature. Values with different letters are significantly different $(p<$ $0.05)$.

$90 \%$ RBO was very weak. The intensity of off-flavor from fish oil was related with the level of volatile compounds such as propanal and acrolein. Most of off-flavor from fish oil during autoxidation could be due to propanal and acrolein, because they are known to be off-flavor compounds from vegetable oils rich in $\alpha$-linolenic acid (18:3) during thermal oxidation ${ }^{11)}$. Reduced propanal and acrolein in fish oil by blending RBO could not give off-flavor. Especially, blending with more than $75 \%$ RBO was effective for improving the flavor stability of fish oil.

Our group tested blending effects of other vegetable oils. However, remarkable stabilization effects which RBO provided were not observed for other vegetable oils from the point view of sensory evaluation. Canola oil produced more 
than $10 \mathrm{ppb}$ acrolein and propanal at least after autoxidation for 28 days (data not shown). One of stabilization effects of RBO is due to diluting effects of RBO. Blending of RBO to fish oil reduced the n-3 PUFA content. The n-3 PUFA content was reduced to $25 \%$ of original fish oil by blending of $75 \% \mathrm{RBO}$. Another one of stabilization effects of RBO may be due to high level of vitamin E. Vitamin E level of RBO was $130 \mathrm{mg} / 100 \mathrm{~g}$ and much higher than other vegetable oils. Especially, RBO had high level of tocotrienols (55 mg/100 g). It is well known that antioxidant activity of tocotrienols is higher than that of corresponding tocopherols $^{13)}$. Therefore, tocotrienols contained in RBO could improve the oxidative and flavor stability of fish oil besides tocopherols. $\gamma$-Oryzanol as an antioxidant ${ }^{14)}$ also could contribute to the stabilization effects of RBO. Moreover, our group found that oleic acid located in triacylglycerol could reduce the oxidation rate of sardine oil rich in n-3 PUFAs ${ }^{15)}$. RBO consisted of $43 \%$ oleic acid and $36 \%$ linoleic acid as major fatty acids. Oleic acid content of fish oil blended with $75 \%$ RBO was $38 \%$ of total fatty acids. Considerable amounts of oleic acid in fish oils blended with more than $75 \%$ RBO may also contribute to their oxidative and flavor stability. From these reasons, blending of RBO may be effective for maintaining the quality of other fish oils during storage.

Even if the contents of n-3 PUFAs such as EPA and DHA in fish oil are reduced by blending with $75 \%$ RBO, residual n-3 PUFAs in blended fish oil will show physiological and nutritional effects to humans.

\section{Conclusions}

When fish oil was blended with RBO and stored at room temperature in the dark, the production of hydroperoxides and volatile compounds during its autoxidation were retarded. Blending of RBO improved the oxidative and flavor stabilities of fish oil. Especially, blending with more than $75 \% \mathrm{RBO}$ was effective for maintaining the flavor quality of fish oil during storage.

\section{References}

1) Simopouls A.P.; Kifer, R.R.; Martin, R.E. Health Effects of Polyunbsaturated Fatty Acids in Sea Foods. Academic Press, Orlando (1986).

2) Lands, W.E.M. Polyunsaturated Fatty Acids and Eicosanoids. American Oil Chemists' Society, Champaign (1987).
3) Vergrosen, A.J. The Role of Fats in Human Nutrition. Academic Press, London (1989).

4) Fujimoto, K. Properties and Physiological Activities of Fish Oils. Koseishakoseikaku, Tokyo (1993).

5) Shiota, M.; Konishi, H.; Tatsumi, K. Oxidative stability of fish oil blended with butter. J. Dairy Sci. 82, 18771881 (1999).

6) Saga, L.C.; Kristinova, V.; Kirkhus, B.; Jacobsen, C.; Skaret, J.; Liland, K.H.; Rukke, E.-O. Characterization of oxidative stability of fish oil- and plant oil-enriched skimmed milk. J. Am. Oil Chem. Soc., 90, 113-121 (2013).

7) Totani, Y. Properties and Application of Fats and Oils. Saiwaishobo, Tokyo (2012).

8) JOCS Standard Method 2.5.2.3.2013, "Fatty acid composition". in Standard Methods for the Analysis of Fats, Oils, and Related Materials (Japan Oil Chemists' Society, ed.), Japan Oil Chemists' Society, Tokyo (2013).

9) JOCS Standard Method 2.4.10-2013, "Tocopherols" . in Standard Methods for the Analysis of Fats, Oils, and Related Materials (Japan Oil Chemists' Society, ed.), Japan Oil Chemists' Society, Tokyo (2013).

10) JOCS Standard Method 2.5.2.1_-2013, "Peroxide value". in Standard Methods for the Analysis of Fats, Oils, and Related Materials (Japan Oil Chemists' Society, ed.), Japan Oil Chemists' Society, Tokyo (2013).

11) Endo, Y.; Hayashi, C.; Yamanaka, T.; Takayose, K.; Yamaoka, M.; Tsuno, T.; Nakajima, S. Linolenic acid as the main source of acrolein formed during heating of vegetable oils. J. Am. Oil Chem. Soc. 90, 959-964 (2013).

12) Endo, Y.; Aoyagi, T.; Fujimoto, K. Effects of the triacylglycerol structure on volatile components produced during eicosapentaenoic acid autoxidation. J. Jpn. Oil Chem. Soc. 47, 873-878(1998).

13) Seppanen, C.M.; Song, Q.; Saari Csallany, A. The antioxidant functions of tocopherol and tocotrienol homologues in oils, fats, and food systems. J. Am. Oil Chem. Soc. 87, 469-481 (2013).

14) Xu, Z.; Hua, N.; Godber, J.S. Antioxidant activity of tocopherols, tocotrienols, and gamma-oryzanol components from rice bran against cholesterol oxidation accelerated by 2,2' -azobis (2-methylpropionamidine) dihydrochloride. J. Agric. Food Chem. 49, 2077-2081 (2001).

15) Endo, Y.; Kimoto, H.; Fujimoto, K. Retarded autoxidation of sardine oil with oleate. Biosci. Biotech. Biochem. 57, 2202-2204(1993). 